\title{
EXISTENCE THEORY FOR NONLINEAR STURM-LIOUVILLE PROBLEMS WITH UNBOUNDED NONLINEARITIES
}

\section{DANiEl MARONCElli AND Jesús RodrígueZ}

Abstract. In this work we provide conditions for the existence of solutions to nonlinear SturmLiouville problems of the form,

$$
\left(p(t) x^{\prime}(t)\right)^{\prime}+q(t) x(t)+\lambda x(t)=f(\varepsilon, x(t))
$$

subject to

$$
a x(0)+b x^{\prime}(0)=0 \text { and } c x(1)+d x^{\prime}(1)=0 .
$$

Our approach will be topological, utilizing both degree theory and the Lyapunov-Schmidt procedure.

Mathematics subject classification (2010): 34B15.

Keywords and phrases: boundary value problems,Sturm-Liouville, Lyapunov-Schmidt procedure, topological degree theory.

\section{REFERENCES}

[1] S. Chow, J.K. Hale, Methods of Bifurcation Theory, Springer, Berlin, 1982.

[2] D.L. Etheridge, J. RodrígueZ, Periodic solutions of nonlinear discrete-time systems, Appl. Anal., 62 (1996), 119-137.

[3] J.K. Hale, Applications of Alternative Problems, Lecture Notes, Brown University, Providence, RI.

[4] J.K. Hale, Ordinary Differential Equations, Robert E. Kreiger Publishing Company, Malabar FL, 1980.

[5] W.G. Kelley, A.C. Peterson, The theory of differential Equations, Springer 2010.

[6] E.M. Landesman, A.C. Lazer, Nonlinear Perturbations of Linear Elliptic Boundary Value Problems at Resonances, J. Math. Mech., 19 (1969), 609-623.

[7] A.C. LAZER, D.F. LEACH, Bounded perturbations of forced harmonic oscillators at resonance, Ann. Mat. Pure Appl., 82 (1969), 49-68.

[8] R. MA, Nonlinear discrete Sturm-Liouville problems at resonance, Nonlinear Anal., 67 (2007), 3050 3057.

[9] D. Maroncelli, J. Rodríguez, On the solvability of nonlinear impulsive boundary value problems, Topol. Methods Nonlinear Anal., accepted.

[10] J. Rodríguez, Nonlinear discrete Sturm-Liouville problems, J. Math. Anal. Appl., 308, Issue 1 (2005), 380-391.

[11] J. RodrígueZ, Z. Abernathy, On the solvability of nonlinear Sturm-Liouville problems, J. Math. Anal. Appl., 387, Issue 1 (2012), 310-319.

[12] J. RodríGUEZ, D.L. ETHERIDGe, Periodic solutions of nonlinear second-order difference equations, Adv. Difference Eqn., 2 (2005), 119-137.

[13] J.F. RodRíGUEZ, Existence theory for nonlinear eigenvalue problems, Appl. Anal., 87 (2008), 293 301.

[14] N. Rouche, J. Mawhin, Ordinary differential equations, Pitman, London, 1980. 